\title{
Outcome of patients with non-Hodgkin's lymphoma of the stomach after gastrectomy: clinicopathologic study and reclassification according to the revised European-American lymphoma classification
}

\author{
Ken-IChi Mafune ${ }^{1}$, Yoichi Tanaka ${ }^{1}$, Yasuo Suda ${ }^{1}$, and Toshiyuki Izumo ${ }^{2}$ \\ ${ }^{1}$ Division of Abdominal Surgery, Saitama Cancer Center Hospital, Saitama, Japan \\ ${ }^{2}$ Division of Clinical Pathology, Saitama Cancer Center Hospital, Saitama, Japan
}

\begin{abstract}
Background. The best treatment for patients with nonHodgkin's lymphoma (NHL) of the stomach is still uncertain. The revised European-American lymphoma (REAL) classification has helped to define new, potentially more appropriate classification schemes for gastric lymphomas.

Methods. Fifty-one resected gastric lymphomas were reclassified according to the REAL classification, and the efficacy of multimodal treatment was examined retrospectively. The principal treatment plan consisted of: (1) surgical resection of the stomach with lymph node dissection, followed by (2) systemic chemotherapy, mainly using the cyclophosphamide/ doxorubicin/vincristine/prednisone (CHOP) regimen.

Results. According to the Ann Arbor classification, 27 patients had stage IE, 19 had stage IIE, and 5 had stage IV NHL. Using the REAL classification, we diagnosed diffuse large Bcell lymphoma (DLBL) in 23 patients, marginal zone B-cell (low-grade mucosa-associated lymphoid tissue [MALT]-type) lymphoma in 22, follicle center lymphoma in 4, mantle cell lymphoma in 1, and peripheral T-cell lymphoma in 1 patient. Nine of the 51 patients relapsed, and 8 patients with DLBL died of cancer. Survival rates at 5 years after surgery were $\mathbf{9 6 . 0} \%$ for stage IE, $83.3 \%$ for stage IIE, and $87.0 \%$ for all patients. Univariate analysis indicated that the tumor histology (according to the REAL classification), depth of invasion, degree of nodal involvement, Ann Arbor staging, and chemotherapy had an impact on patient outcome $(P=0.0018 ; P=$ 0.0002; $P=0.0308 ; P=0.0016$, and $P=0.0118$, respectively). Conclusions. These data reveal that gastric NHL, especially of the low-grade MALT-type, often remains localized and has a good prognosis after surgery. The REAL classification was useful for classifying new categories of NHL, including the MALT-type, in the clinical setting, and for determining the optimal treatment modality for gastric NHL.
\end{abstract}

Offprint requests to: $\mathrm{K}$. Mafune

Department of Gastrointestinal Surgery, the University of Tokyo Graduate School of Medicine, 7-3-1 Hongo, Bunkyoku, Tokyo 113-8655, Japan

Received: December 11, 2000 / Accepted: July 18, 2001
Key words Gastric non-Hodgkin's lymphoma - The revised European-American lymphoma classification · Marginal zone B-cell lymphoma - Mucosa-associated lymphoid tissue-type lymphoma

\section{Introduction}

Approximately 5\% of all primary non-Hodgkin's lymphomas (NHL) are located in the gastrointestinal tract $[1,2]$, and the stomach is the most common site of extranodal NHL [3]. The incidence of primary gastric NHL has doubled within the past 10 years [4]; however, the definition of this type of tumor remains a matter of debate, because the stomach is more often secondarily involved in the dissemination of nodal lymphomas.

To date, the main treatment modality has been surgical resection, while the role of adjuvant therapy has remained poorly defined. However, although primary gastric lymphomas are resectable, several studies have documented that nonsurgical treatment has yielded reasonable outcomes [2,5]. This dilemma over management is further compounded by reports of massive hemorrhage or perforation of nonresected, medically treated lesions. The infrequency of gastric NHL makes it difficult to evaluate therapeutic results and discourages the conducting of prospective trials.

In 1984, Isaacson and Wright [6] introduced the new concept of extranodal lymphomas arising from mucosaassociated lymphoid tissue (MALT), and classification schemes for gastric lymphomas had to be changed. The revised European-American lymphoma (REAL) classification was introduced in 1994 [7], and has helped to define new, potentially more appropriate classification schemes for gastric lymphomas. We therefore reclassified gastric lymphomas previously resected at the Saitama Cancer Center according to the REAL classification, and here discuss their treatment outcomes. 


\section{Subjects and methods}

Fifty-one patients with gastric lymphoma underwent gastrectomy at our division of the Saitama Cancer Center from January 1981 to July 1997. According to the criteria proposed by Dawson et al. [8], the lesions were all primary to the stomach; patients with secondary gastric lymphoma were excluded from the study. Clinicopathologic data, such as age, sex, tumor location, tumor number, tumor gross type, depth of tumor invasion, degree of lymph node involvement, and clinical staging were obtained from the patients' hospital records. Recent follow-up information was obtained for all patients either from their outpatient records or by contacting the patient or a close relative.

\section{Depth of invasion and degree of lymph node involvement}

The depth of invasion and degree of lymph node involvement were classified according to the 1987 version of the TNM staging criteria for gastric cancer [9]. T staging was modified slightly according to the Japanese classification of gastric carcinoma [10] as follows: T1, tumor invades the lamina propria or submucosa; T2MP, tumor invades the muscularis propria; T2-SS, tumor invades the subserosa; T3, tumor penetrates the serosa, no invasion of adjacent structures; T4, tumor invades adjacent structures. $\mathrm{N}$ staging was classified as follows: N0, negative nodes; N1, positive nodes among the perigastric nodes within $3 \mathrm{~cm}$ of the primary tumor; $\mathrm{N} 2$, positive nodes among the perigastric nodes more than $3 \mathrm{~cm}$ from the primary tumor or along the hepatic, left gastric, splenic or celiac arteries; N3, positive nodes among the hepatoduodenal, paraaortic or distant abdominal nodes; N4, positive nodes beyond N3.

\section{Clinical staging and histologic classification}

The lesions were staged according to the Ann Arbor staging system criteria for Hodgkin's lymphoma [11], modified for NHL of the stomach. These lesions were then histologically reclassified according to the REAL classification criteria [7].

\section{Treatment modalities}

The principal treatment plan for gastric lymphoma at the Saitama Cancer Center consists of: (1) surgical resection of the stomach (either partial or total gastrectomy) with lymph node dissection, followed by (2) systemic chemotherapy for patients with nodal involvement, mainly using the cyclophosphamide/doxorubicin/ vincristine/prednisone (CHOP) regimen, with (3) no irradiation. Chemotherapy is also performed if the lym- phoma is not completely resected or if the tumor recurs during the follow-up period.

The CHOP regimen used as the principal chemotherapy protocol consists of: cyclophosphamide, $750 \mathrm{mg} / \mathrm{m}^{2}$ intravenously on day 1 ; doxorubicin, $50 \mathrm{mg}$ / $\mathrm{m}^{2}$ intravenously on day 1 ; vincristine, $1.4 \mathrm{mg} / \mathrm{m}^{2}$ intravenously on day 1 ; and prednisone, $100 \mathrm{mg} / \mathrm{m}^{2}$ orally on days $1-5$. Cycles are repeated at 21- to 28-day intervals, and the patients participating in this study received a mean total of 7 cycles (range, 1-16 cycles).

\section{Statistical methods}

The $\chi^{2}$ test was used to analyze discrete clinical variables, and Fisher's exact test was applied when the number of observations was small. For 46 patients (excluding 5 who died of unrelated diseases), survival data were calculated by the Kaplan-Meier actuarial method, and various subgroups and their therapeutic results were compared to identify potential prognostic factors that might have an effect on treatment outcome. Significant differences between the calculated survival curves were identified by univariate analysis, using the Cox-Mantel test. $P$ values less than 0.05 were considered statistically significant.

\section{Results}

\section{Tumor staging}

The 51 patients with gastric NHL were classified according to the Ann Arbor system. According to this classification, 27 had stage IE, 19 had stage IIE, and 5 had stage IV disease. The $46(90.2 \%)$ patients classified as having stage IE or IIE disease were suitable for surgery. The 5 stage IV patients were not really suitable for surgery, but 1 underwent gastrectomy because of gastric perforation that occurred during chemotherapy.

\section{Treatment modalities}

Treatment modalities were selected according to the principal treatment plan of the Saitama Cancer Center. However, treatment methods varied according to the extent of the disease, the patient's clinical condition, and the preference of the attending physicians. The treatments actually performed were: (1) surgical resection of the stomach $(n=51)$ with lymph node dissection $(n=50)$, followed by (2) systemic chemotherapy $(n=$ $26)$, mainly using the CHOP regimen $(n=22)$, with (3) no irradiation. The non-CHOP chemotherapy regimens used were mostly developed for gastric cancer, and seemed ineffective against gastric lymphoma.

Chemotherapy was given in addition to surgery in 7 of the 27 stage IE patients, 15 of the 19 stage IIE 
patients, and 2 of the 5 stage IV patients. The CHOP regimen was used in 4 of the 27 stage IE patients, 15 of the 19 stage IIE patients, and 3 of the 5 stage IV patients. At our hospital, adjuvant chemotherapy is not normally used for stage IE gastric lymphoma, i.e., when the tumor is limited to the stomach and there is no nodal involvement. Therefore, chemotherapy was added to surgery significantly more often for stage IIE patients than for stage IE patients $(P=0.0080)$.

As regards surgical therapy, total gastrectomy was not always performed if the lesion was confined to the stomach. Total gastrectomy was performed in 41 patients $(80.4 \%)$, while partial (distal) gastrectomy was performed in the remaining 10 (9 of the 27 stage IE patients and 1 of the 19 stage IIE patients). Distal gastrectomy was performed significantly more often in stage IE patients $(P=0.0124)$. Of the 41 patients who underwent total gastrectomy, the spleen was coresected in 37 . In 12 of these 37 patients ( 3 of 17 with stage IE, 6 of 15 with stage IIE, and 3 of 5 with stage IV disease) the pancreatic tail was also coresected to achieve complete D2 dissection. Lymph node dissection was performed, as for gastric cancer, in 50 patients. In 48 of the 51 patients, $\mathrm{D} 2$ or more lymph node dissection was performed.

\section{Histologic classification}

When the tumors were reclassified according to the REAL system, diffuse large B-cell lymphoma (DLBL) was diagnosed in 23 patients, marginal-zone B-cell lymphoma (MZBL) in 22, follicle center lymphoma (FCL) in 4, mantle cell lymphoma (MCL) in 1, and peripheral T-cell lymphoma (PTL) in 1.

There were 22 low-grade MALT-type lymphomas in the 51 patients with gastric NHLs $(43.1 \%)$ and all were classified as MZBLs. Six (27.3\%) of the 22 patients with MZBLs had lymph node involvement, and were classified as stage IIE. Compared with the frequency of other histologic types of lymphomas, MZBLs occurred significantly more often in less advanced disease stages $(P=$ $0.0240)$, especially in stage I $(P=0.0137)$. All stage IV tumors were classified as DLBL (Table 1). In 16 $(72.7 \%)$ of the 22 patients with MZBL (low-grade MALT-type), the depth of invasion was T1 (within the submucosal layer), and in $19(86.4 \%)$ it was within the proper muscle layer (T1 or T2-MP). Compared with the frequency of other histologic types of lymphoma, MZBL occurred significantly more often within the proper muscle layer $(P=0.0155)$ (Table 2$)$.

In 25 of the 27 stage IE patients, 8 of the 19 stage IIE patients, and 1 of the 5 stage IV patients, the tumor remained within the subserosal layer (T1 or T2). In 25 of the $27 \mathrm{~N} 0$ patients, 5 of the $11 \mathrm{~N} 1-2$ patients, and 4 of 13 N3-4 patients, the tumor also remained within the
Table 1. Tumor staging and histologic types of NHL according to the REAL classification

\begin{tabular}{lrrrrrr}
\hline Stage & DLBL & MZBL & FCL & MCL & PTL & Total \\
\hline IE & 8 & 16 & 2 & 0 & 1 & 27 \\
IIE & 10 & 6 & 2 & 1 & 0 & 19 \\
IV & 5 & 0 & 0 & 0 & 0 & 5 \\
Total & 23 & 22 & 4 & 1 & 1 & 51 \\
\hline
\end{tabular}

NHL, Non-Hodgkin's lymphoma; REAL, revised EuropeanAmerican lymphoma; DLBL, diffuse large B-cell lymphoma; MZBL, marginal-zone B-cell lymphoma; FCL, follicle center lymphoma; MCL, mantle cell lymphoma; PTL, peripheral T-cell lymphoma

Table 2. Depth of invasion and histologic types of NHL

\begin{tabular}{lrrrrrr}
\hline & & \multicolumn{3}{c}{ T2 } & & \\
\cline { 3 - 5 } Histology & T1 & MP & SS & T3 & T4 & Total \\
\hline DLBL & 7 & 2 & 2 & 4 & 8 & 23 \\
MZBL & 16 & 3 & 0 & 2 & 1 & 22 \\
FCL & 1 & 0 & 2 & 1 & 0 & 4 \\
MCL & 0 & 0 & 0 & 1 & 0 & 1 \\
PTL & 1 & 0 & 0 & 0 & 0 & 1 \\
Total & 25 & 5 & 4 & 8 & 9 & 51 \\
\hline
\end{tabular}

DLBL, diffuse large B-cell lymphoma; MZBL, marginal-zone B-cell lymphoma; FCL, follicle center lymphoma; MCL, mantle cell lymphoma; PTL, peripheral T-cell lymphoma; MP, proper muscle layer; SS, subserosal layer

subserosal layer. Therefore, the depth of tumor invasion correlated with the Ann Arbor tumor stage $(P=$ $0.0001)$ and with the degree of lymph node involvement $(P=0.001)$.

\section{Patient outcomes}

Nine of the 51 patients relapsed, and 8 died of their cancer. Six died within 14 months of surgery, despite having received postoperative chemotherapy. Two died more than 5 years after surgery. For 46 patients (excluding 5 who died of unrelated diseases), the survival rate at 5 years after surgery was $87.0 \%$ (Fig. 1A), being $96.0 \%$ for stage IE patients and $83.3 \%$ for stage IIE patients (Fig. 1B).

The results of the univariate analysis to determine predictors of survival are shown in Table 3. The tumor histology, depth of invasion, degree of nodal involvement, Ann Arbor staging, and chemotherapy had an impact on patient outcome $(P=0.0018 ; P=0.0002$; $P=0.0308 ; P=0.0016$, and $P=0.0118$, respectively). However, because tumor stage was correlated with other factors in this study, these may not be independent predictors of survival.

Patients in stage IE had a significantly better outcome than those in stage IIE or IV $(P=0.0124$ and $P<$ 0.0001 , respectively) (Fig. 1B, Table 3). Survival rates at 


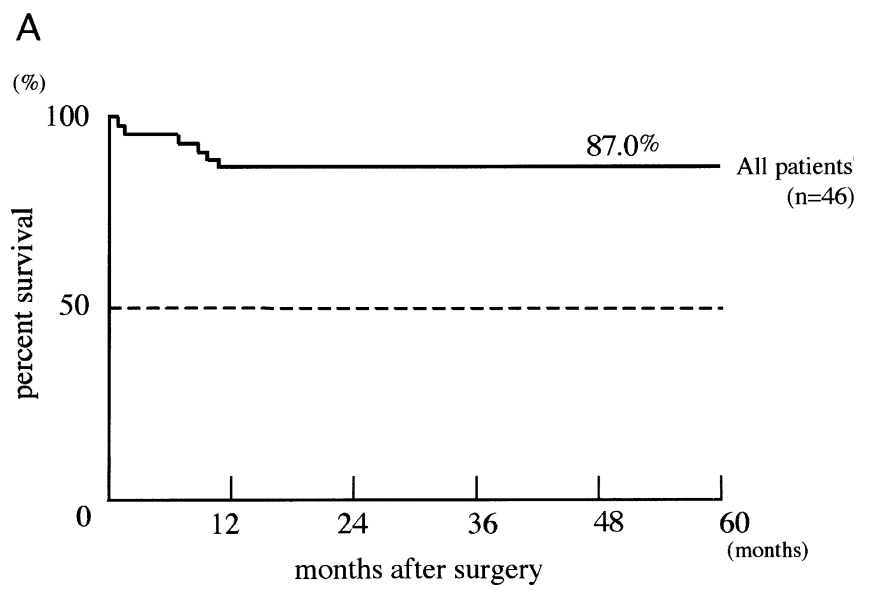

B

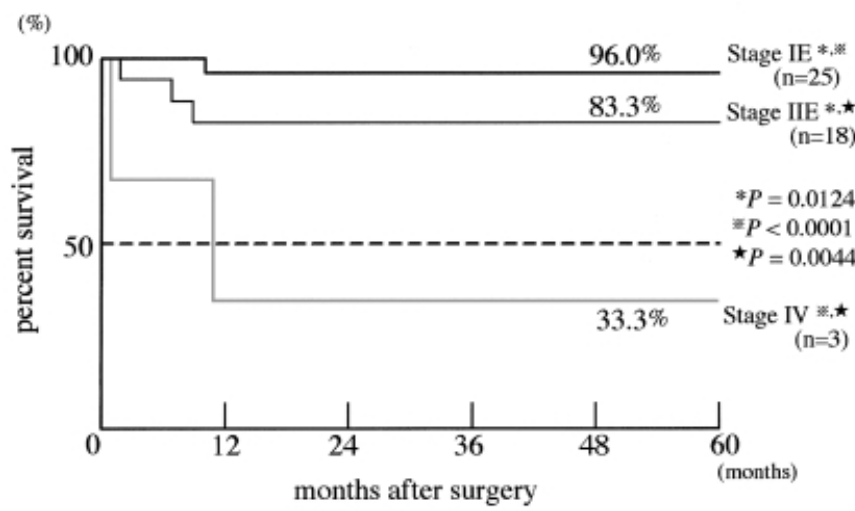

C

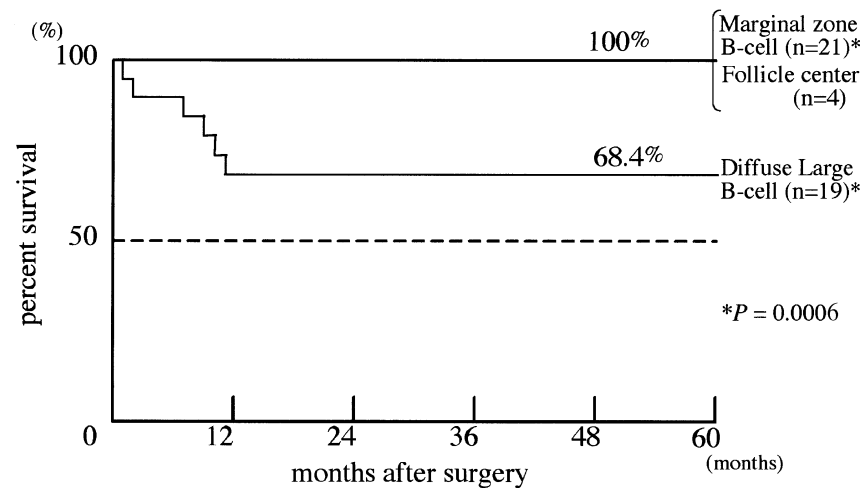

Fig. 1. A Kaplan-Meier survival curve for all patients with gastric non-Hodgkin's lymphoma (NHL) after gastrectomy. B Kaplan-Meier survival curves for patients with gastric NHL, classified by the Ann Arbor staging system. C KaplanMeier survival curves for patients with gastric NHL, classified by the revised European-American lymphoma (REAL) system: i.e., marginal-zone B-cell (MZBL), follicle center, or diffuse large B-cell lymphomas. The probability of survival was significantly greater in patients with MZBL than in patients with diffuse large B-cell lymphoma. D Kaplan-Meier survival curves for patients with gastric NHL, classified by depth of
D

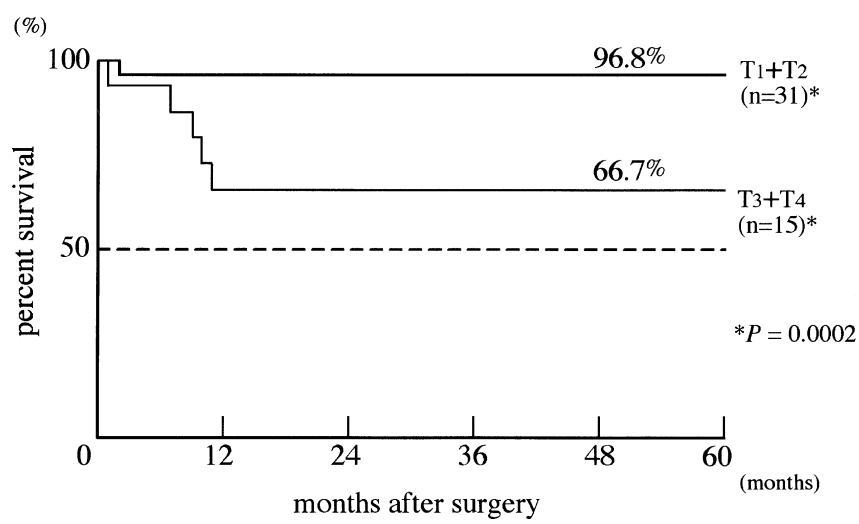

E

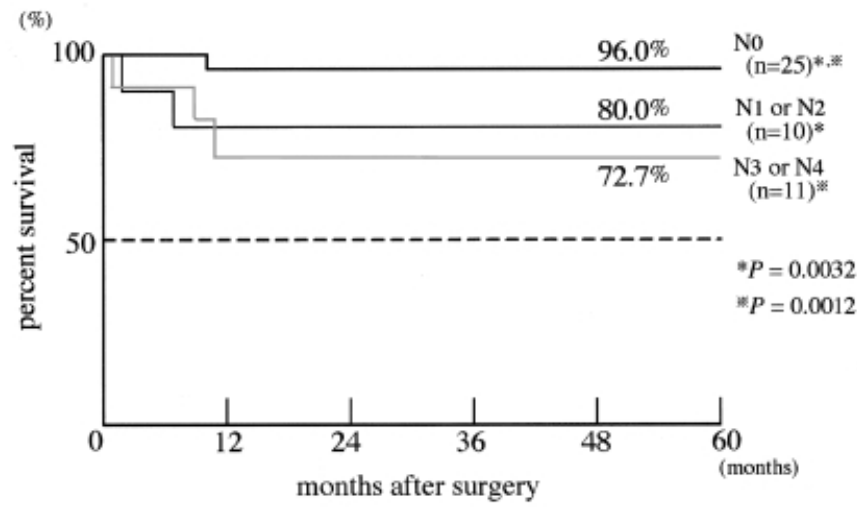

invasion: i.e., $\mathrm{T} 1$ or $\mathrm{T} 2$ (tumor confined within the subserosa), and T3 or T4 (tumor penetrating through the serosa). The probability of survival was significantly greater when the tumor remained within the subserosa. E Kaplan-Meier survival curves for patients with gastric NHL, classified by degree of lymph node involvement, i.e., N0 (without nodal involvement), N1 or N2 (with regional lymph node involvement), and $\mathrm{N} 3$ or N4 (with spread beyond the regional nodes). The probability of survival was significantly greater in patients without lymph node metastasis 
Table 3. Results of univariate analyses to identify predictors of survival in patients with gastric non-Hodgkin's lymphoma

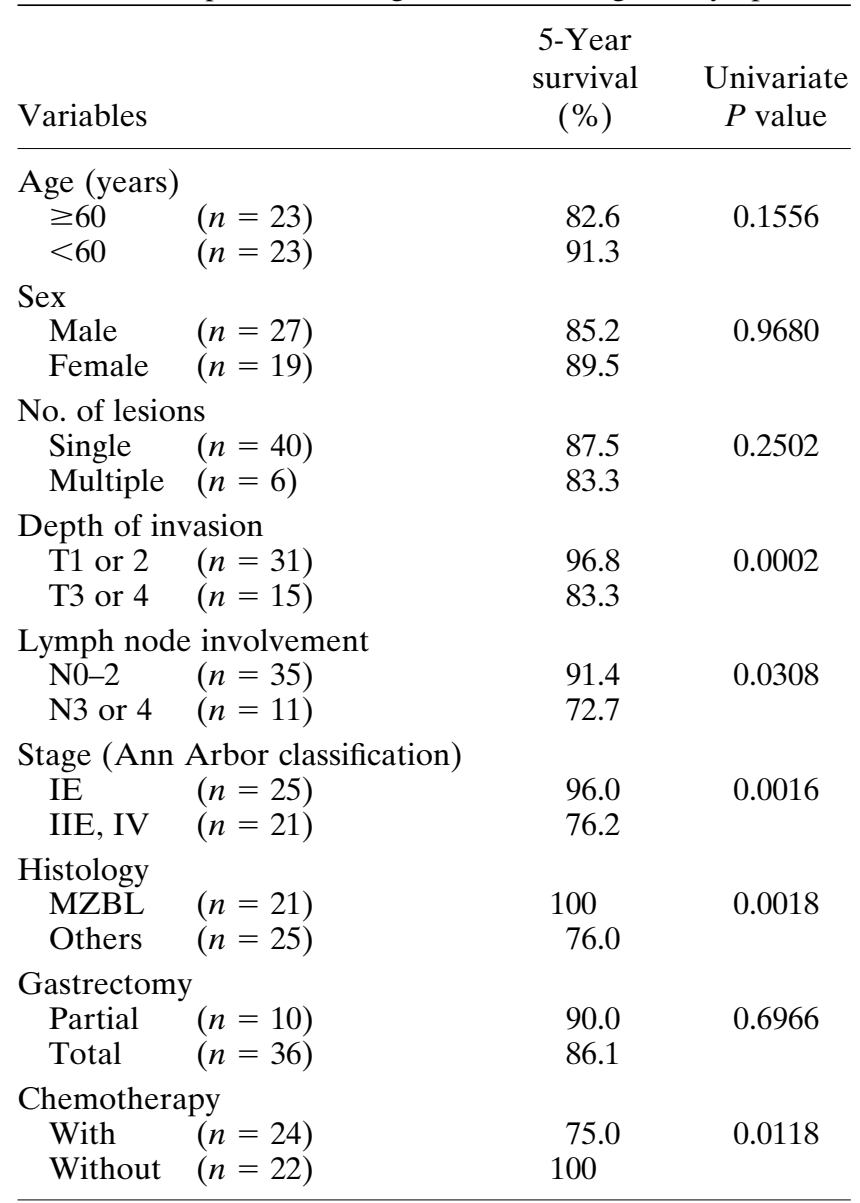

MZBL, Marginal zone B-cell lymphoma

5 years after surgery were $100 \%$ for MZBLs or follicle center lymphomas and $68.4 \%$ for diffuse large B-cell lymphomas, and patients with MZBL had a significantly better outcome than those with DLBL $(P=0.0006)$ (Fig. 1C). Univariate analysis also showed that patients with MZBL (low-grade MALT-type) had a significantly better outcome than patients with other types of NHL $(P=0.0018)$ (Table 3$)$. While one of the patients with MZBL relapsed after surgery, complete response was observed after chemotherapy. In contrast, patients with DLBL had a significantly worse outcome $(P=$ $0.0220)$. Patients with lymphoma confined within the subserosal layer (T1 or 2) had a significantly better outcome than those whose tumors penetrated through the serosa (T3 or 4; $P=0.0002$ ) (Fig. 1D). Patients without nodal involvement (N0) had a significantly better outcome than those with regional lymph node involvement (N1 or N2) and those with spread beyond the regional nodes $(\mathrm{N} 3$ or N4) $(P=0.00032$ and $P=$ 0.0012 , respectively) (Fig. 1E). However, there was no significant difference between patients with $\mathrm{N} 1$ or N2 involvement and patients with $\mathrm{N} 3$ or N4 involvement $(P=0.5754)$.

\section{Discussion}

Various standard systems, such as Rappaport's [12], the Kiel system [13], and the National Cancer Institute's working formulation [1], have been proposed for the histological classification of lymphomas, and have been adopted for extranodal lymphomas such as gastric lymphoma. Although these systems have served well for a long time, they have become increasingly unable to deal with modern concepts of immunology and with newly described clinicopathologic entities. An example of an important, recently recognized clinical entity is the lowgrade lymphoma, MALT. Since its recognition, classification schemes for gastric lymphomas have had to be changed completely, because almost one-third of gastric lymphomas can be classified as MALT types (such as the MZBL discussed in this study). Our results confirm previous reports that most patients with gastric MALT-type lymphoma achieve long-term remission. The MALT concept offers several reasons, stemming from the special biological features of MALT lymphomas, to explain this favorable clinical outcome. These include the homing phenomenon and the tendency of MALT lymphomas to differentiate and regress [6,14-16]. Moreover, molecular genetic investigations support the theory that lymphomas derived from MALT are essentially different from nodal lymphomas, in spite of their morphological similarities $[17,18]$. Therefore, past attempts to classify MALT lymphomas with the standard systems (Rappaport, Kiel, and the National Cancer Institute's working formulation) were unproductive [19].

The REAL classification is suitable for any kind of lymphoma, even though extranodal lymphomas differ from nodal ones and MALT lymphomas differ from other types of extranodal NHL. The REAL classification describes several clinical entities that have been recognized in recent years and cannot easily be identified in the National Cancer Institute's working formulation. Rosenberg [20] criticized the REAL classification on the grounds that it appeared needlessly complex and lacked the clinical underpinnings of earlier systems. He also considered this proposed system, as detailed and helpful as it is for a pathologist, to be inadequate from a clinician's point of view, because all 19 authors of the proposal were pathologists. However, the introduction of the MALT concept has revolutionized lymphoma classification, and earlier standard systems need to be changed dramatically in consequence. According to the REAL classification, low-grade MALT lymphoma can clearly be classified as MZBL. Our present data show the usefulness of the REAL system for classifying gas- 
tric NHL in the clinical setting, even though the system was proposed for use in pathology. Therefore, we believe the REAL classification to be a more appropriate standard system for gastric NHL classification.

At the same time as the REAL classification was introduced, a new, simple classification of gastrointestinal lymphoma was introduced [21], in which MALT lymphoma was classified into two grades, low and high. This system, a pathologic classification of gastrointestinal lymphoma, was based on the updated Kiel classification [22] which had been modified to adopt new categories of NHL. The high-grade MALT lymphoma in this classification [21] is biologically different from the low-grade MALT lymphoma. However, the same name, MALT, is sometimes confusing for clinicians in terms of determining the optimal treatment modality. According to the REAL classification, the high-grade MALT-type lymphoma was classified as DLBL in our present study. Our data showed that about $90 \%$ of gastric NHLs were classified as DLBL or MZBL, similar to high- or lowgrade lymphoma. These two classifications were made according to the same concept, consequently, they seem to be the same. Therefore, the REAL classification was really simple, not as complicated as Rosenberg [20] indicated, when this classification was used for gastric NHL. Moreover, we showed that the REAL classification was correlated with the Ann Arbor stage, depth of invasion, and degree of lymph node metastasis. Thus, the REAL classification is useful not only for pathologists but also for clinicians like us. However, there is no doubt that current classification systems, including the REAL classification, must be slightly modified for gastrointestinal lymphomas.

In our study, the Ann Arbor staging system [11] was initially used for the clinical staging of gastric NHLs. This system was originally designed for Hodgkin's lymphoma, but is commonly applied to non-Hodgkin's lymphoma. In a review of published data from 100 series of gastric lymphoma patients, the most common grading system used was based upon the Ann Arbor classification [23]. However, we consider that this system is not entirely suitable for classifying primary gastric lymphomas, which seldom involve the upper side of the diaphragm. Furthermore, the incidence of stage IIIE disease is quite low. Stage IIE can be divided into two substages, as in Musshoff's modification [24]: stage IIE1, tumor in the stomach with spread to contiguous lymph nodes; and stage IIE2, tumor in the stomach with spread to lymph nodes that are noncontiguous with the primary tumor. This more precise subclassification may be required in stage IIE disease, because regional radicality can be achieved by performing lymph node dissection in stage IIE1 patients, but not in stage IIE2 patients. However, in our series, patients with $\mathrm{N} 1$ or N2 nodal involvement did not have a better prognosis than patients with $\mathrm{N} 3$ or N4 nodal involvement. This finding implies that Musshoff's modification may not be required as a predictor of survival, and this also suggests that lymph node dissection may not have an important impact on the patient. The outcome for patients with NHL involving the lymph nodes (stage IIE or greater) thus seems to depend on the efficacy of the adjuvant chemotherapy regimen, and this may be the optimal role for chemotherapy in the treatment of gastric NHL.

A major deficit of the Ann Arbor staging system is the lack of variables to describe the extent of the primary tumor and the depth of tumor invasion, which have been reported to be of prognostic significance in many studies, including ours [2,25-29]. Naqvi et al. [30] developed guidelines for predicting prognosis by grading lymphoma of the gastrointestinal tract into four stages, taking into consideration the extent of the primary tumor, the presence of lymph node involvement, invasion of adjacent structures or the presence of perforation, and distant metastasis. This was a reasonable clinical staging system because it took into account both $\mathrm{T}$ and $\mathrm{N}$ factors. However, in this classification, penetration through the serosa was not considered a predictor of survival (which it was in our study), whereas invasion of adjacent structures and perforation were important factors. In 1994, Shimodaira et al. [29] proposed a staging system based on the principles of the TNM system. The $T$ variable was classified into five stages based on the depth of invasion, with invasion of adjacent organs included in the pattern of nodal spread. In our study, we classified the depth of invasion into four stages according to the TNM system for gastric cancer [9], with a slight modification according to the Japanese classification of gastric carcinoma [10]. Our T-staging system was, therefore, similar to that proposed by Shimodaira et al. [29]. Our results suggested that there was no need to separate the proper muscle layer (T2 MP, or Shimodaira's T2) and the subserosal layer (T2 SS, or Shimodaira's T3) within the grading system. However, separating T2 and T3 (Shimodaira's T3 and T4) is important, because our results indicated that patients with lymphoma confined within the subserosal layer had a significantly better outcome than those whose tumor penetrated through the serosa, and the proposal of Shimodaira and colleagues was based on similar results. These findings suggest that a simpler classification of the depth of tumor invasion than that of our T-staging system may be useful for gastric NHL. In the older version of the TNM system for gastric cancer that we used, the N-staging classification is similar to that in the modified Ann Arbor system. We believe that a simpler TNM staging system, based on the Ann Arbor system, could be proposed after more patients have been studied. 


\section{Conclusions}

The REAL classification proved useful for classifying new categories of NHL, in particular MZBL (low-grade MALT type), in the clinical setting. Our data showed that gastric NHL, in particular, MZBL, often remains localized and has a good prognosis after surgery.

\section{References}

1. The non-Hodgkin's lymphoma pathologic classification project. National Cancer Institute sponsored study of classifications of non-Hodgkin's lymphomas. Summary and description of a working formulation for clinical usage. Cancer 1982;49:2112-35.

2. Brooks J, Enterline HT. Primary gastric lymphomas. A clinicopathologic study of 58 cases with long-term follow-up and literature review. Cancer 1983;51:701-11.

3. Otter R, Bieger R, Kluin PM, Hermans J, Willemze R. Primary gastrointestinal non-Hodgkin's lymphoma in a population-based registry. Br J Cancer 1989;60:745-50.

4. Severson RK, Davis S. Increasing incidence of primary gastric lymphoma. Cancer 1990;66:1283-7.

5. Maor MH, Maddux B, Osborne BM, Fuller LM, Sullivan JA, Nelson RS, et al. Stage IE and IIE non-Hodgkin's lymphomas of the stomach: comparison of treatment modalities. Cancer 1984; 54:2330-7.

6. Isaacson $\mathrm{P}$, Wright $\mathrm{DH}$. Malignant lymphoma of mucosa-associated lymphoid tissue. A distinctive type of B-cell lymphoma. Cancer 1984;52:1410-6.

7. Harris NL, Jaffe ES, Stein H, Banks PM, Chan JKC, Cleary ML, et al. A revised European-American classification of lymphoid neoplasms: a proposal from the International Lymphoma Study Group. Blood 1994;84:1361-92.

8. Dawson IMP, Cornes JS, Morson BC. Primary malignant lymphoid tumours of the intestinal tract. Report of 37 cases with a study of factors influencing prognosis. Br J Surg 1961;49:809.

9. Hermanek P, Sobin LH. International Union against Cancer. TNM classification of malignant tumors. 4th ed. New York Tokyo Heidelberg Berlin: Springer-Verlag; 1987.

10. Japanese Gastric Cancer Association. Japanese classification of gastric carcinoma. 2nd English ed. Gastric Cancer 1998;1:10-24.

11. Carbone PP, Kaplan HS, Musshoff K, Smithers DW, Tubiana M. Report of the committee on Hodgkin's staging classification. Cancer Res 1971;31:1860-1.

12. Rappaport H. Tumors of the hematopoietic system. In: Atlas of tumor pathology, section 3, fascicle 8. Washington DC: U.S. Armed Forces Institute of Pathology; 1966.

13. Lennert K, Mohri N, Stein H, Kaiserling E. The histopathology of malignant lymphoma. Br J Haematol 1975;39(Suppl):193-203.

14. Isaacson PG, Spencer GJ, Wright DH. Classifying primary gut lymphomas. Lancet 1988;11:1148-9.

15. Stolte M. Helicobacter pylori gastritis and gastric MALT lymphoma. Lancet 1992;339:745-6.

16. Wotherspoon A, Doglioni C, Diss T, Pan L, Moschini A, de Boni $\mathrm{M}$, et al. Regression of primary low-grade B-cell gastric lymphoma of mucosa-associated lymphoid type after eradication of Helicobacter pylori. Lancet 1993;342:575-7.

17. Horsman D, Gascoyne R, Klasa R, Coupland R. t(11;18) (q21;21.1): A recurring translocation in lymphomas of mucosaassociated lymphoid tissue (MALT)? Genes Chromosom Cancer 1992;4:183-7.

18. Nizze H, Cogliatti SB, von Schilling C, Feller AC, Lennert K. Monocytoid B cell lymphoma: morphological variants and relationship to low-grade B-cell lymphoma of the mucosa-associated lymphoid tissue. Histopathology 1991;18:403-14.

19. Aisenberg AC. Coherent view of non-Hodgkin's lymphoma. J Clin Oncol 1995;13:2656-75.

20. Rosenberg SA. Classification of lymphoid neoplasms. Blood 1994;84:1359-60.

21. Rahatiner A, d'Amore F, Coiffer B, Crowther D, Gospodarowicz $\mathrm{M}$, Isaacson $\mathrm{P}$, et al. Report on a workshop convened to discuss the pathological and staging classifications of gastrointestinal tract lymphoma. Ann Oncol 1994;5:397-400.

22. Stansfeld AG, Diebold J, Kapanci Y, Kelenyi G, Lennert K, Mioduszewska $\mathrm{O}$, et al. Updated Kiel classification for nonHodgkin's lymphoma. Lancet 1988;I:292-3.

23. Brands F, Monig SP, Raab M. Treatment and prognosis of gastric lymphoma. Eur J Surg 1997;163:803-13.

24. Musshoff K. Klinische stadieneinteilung der nicht-Hodgkinlymphoma. Strahlentherapie 1977;153:218-21.

25. Lim FE, Hartman AS, Tan EG, Cady B, Meissner WA. Factors in the prognosis of gastric lymphoma. Cancer 1977;39:1715-20.

26. Shimm DS, Desoretz DE, Anderson T, Linggood RM, Harris NL, Wang CC. Primary gastric lymphoma: an analysis with emphasis on prognostic factors and radiation therapy. Cancer 1983;52: 2044-8.

27. Cogliatti SB, Schmid U, Schumacher U, Eckert F, Hansmann M-L, Hedderich J, et al. Primary B-cell gastric lymphoma: a clinicopathological study of 145 patients. Gastroenterology 1991; 101:1159-70.

28. Radaszkiewicz T, Dragosics B, Bauer P. Gastrointestinal malignant lymphoma of the mucosa-associated lymphoid tissue: factors relevant to prognosis. Gastroenterology 1992;102:1628-38.

29. Shimodaira M, Tsukamoto $Y$, Niwa $Y$, Goto $H$, Hase S, Hayakawa T, et al. A proposed staging system for primary gastric lymphoma. Cancer 1994;73:2709-15.

30. Naqvi MS, Burrows L, Kark AE. Lymphoma of the gastrointestinal tract: prognostic guides based on 162 cases. Ann Surg 1969; 170:221-31. 